\title{
Field Test and Fading Measurement of a Distributed Acoustic Sensor System over a 50 km-long Fiber
}

\author{
Faruk Uyar ${ }^{\mathrm{a}, \mathrm{b}}$, Tolga Kartaloglu ${ }^{\mathrm{b}}$, Ibrahim Ozdur ${ }^{\mathrm{b}, \mathrm{c}}$ and Ekmel Ozbay ${ }^{\mathrm{a}, \mathrm{b}, \mathrm{d}}$ \\ aDepartment of Electrical and Electronics Engineering, Bilkent University, Ankara, Turkey \\ ${ }^{b}$ Nanotechnology Research Center, Bilkent University, Ankara, Turkey \\ ${ }^{c}$ Department of Electrical and Electronics Engineering, Abdullah Gul University, Kayseri, Turkey \\ ${ }^{\mathrm{d}}$ Department of Physics, Bilkent University, Ankara, Turkey
}

\begin{abstract}
In this study, we present a direct detection distributed acoustic sensor based on phase-sensitive optical time domain reflectometer ( $\varphi$-OTDR) with long sensing range and high signal-to-noise ratio (SNR), which is field-tested over a $50 \mathrm{~km}$ long fiber. Due to the random nature of Rayleigh backscattered light and fading phenomena, it is hard to characterize the performance of the system. For this reason, the performance of our sensor is specified in a statistical manner in which the mean SNR is determined using the histograms of the SNR. The SNR values are measured for identical acoustic signals in five different days, total of 48 hours and the SNR histograms are obtained for fiber distances of $100 \mathrm{~m}, 12 \mathrm{~km}, 21 \mathrm{~km}, 30$ $\mathrm{km}, 40 \mathrm{~km}$ and $50 \mathrm{~km}$. The system is field-tested using external disturbances that are generated from a $50-\mathrm{Hz}$ vibrator. The SNR values are extracted from the power spectral density (psd) of the collected data over the monitored fiber span. Our results show that the $\varphi$-OTDR system exhibits a mean SNR of $22.5 \mathrm{~dB}$ at $50 \mathrm{~km}$ distance.
\end{abstract}

Keywords: Distributed acoustic sensor, optical time domain reflectometer, $\varphi$-OTDR, Rayleigh scattering, fading.

\section{INTRODUCTION}

$\varphi$-OTDR based distributed acoustic sensing (DAS) systems have attracted increasing attention in recent years due to its remarkable advantages in a wide range of industrial applications including health monitoring and security of civil infrastructures, railways, oil and gas pipelines, borders, and more [1]. They measure vibrations and detect perturbations along a section of fiber. Different approaches have been adopted to realize the $\varphi$-OTDR system and several efforts have been made to improve the performance. These methods include coherent detection for increased sensitivity [2], hybrid distributed amplification for longer range [3,4], phase demodulation based on phase generated carrier algorithm, 3 x 3 demodulation method, frequency division multiplexing, and digital coherent detection schemes for increased linearity and SNR [5-8]. However, regardless of the interrogation scheme, it is hard to characterize the performance of the system due to the random nature of multipoint interference of Rayleigh backscattered light and fading phenomenon [9]. Since fiberoptic DAS technologies are based on coherent interference of Rayleigh backscattered light from discrete scatterers within the pulse duration, they experience signal fading, which includes interference fading and polarization fading [9]. The former is caused by randomness of Rayleigh scattering and is one of the most important factors that limit the performance. It also raises difficulties in specifying the system performance, particularly SNR. Different techniques were implemented in order to have a reduced interference fading and relatively stable phase sensitivity [10-12]. Additionally, many studies were done to overcome the polarization fading such as adoption of polarization diversity scheme [13], interrogation with orthogonal-state of polarization pulse pair [14] and polarization-maintaining configuration [9]. Even though fading induced noise effects were remarkably mitigated in these studies, the system still suffers from fading behaviors and random fluctuations. These are even more observable in the field tests where the environmental factors such as temperature, humidity as well as soil hardness become relevant. Therefore, considering the randomness of the system, SNR should be statistically presented instead of a single value in order to provide a more complete and indicative figure of merit for the sensor. In the literature, limited attention was given to the statistical parameters such as mean SNR and the variance, to define the performance characteristics of the system. In a recent study, by taking into consideration the random characteristics of the system, a new figure of merit (mean SNR) was introduced to characterize and compare DAS systems

Fiber Optic Sensors and Applications XV, edited by Alexis Mendez, Christopher S. Baldwin, Henry H. Du, Proc. of SPIE Vol. 10654, 106540D · C 2018 SPIE · CCC code:

0277-786X/18/\$18 - doi: $10.1117 / 12.2304735$

Proc. of SPIE Vol. 10654 106540D-1 
$[15,16]$. However, the analysis is limited to laboratory tests and coherent detection schemes; in this manuscript we extend the analysis to field tests and direct detection systems. In this work we experimentally show the statistical characteristics of the SNR of a direct-detection DAS system, calculated over five days and 48 hours of data acquired from the field. We also demonstrate the distribution patterns of SNR in different distances and the change in mean SNR over distance.

\section{BASIC PRINCIPLE}

We first review the basic principle of a $\varphi$-OTDR before assessing the performance of our DAS system. In the $\varphi$-OTDR technique, repeated optical pulses from a highly coherent light source are injected into the sensing fiber. As the pulse propagates along the fiber, the Rayleigh backscattered light waves interfere with each other within the pulse duration. The coherent superposition of the light scattered from randomly placed scatterers yields a $\varphi$-OTDR trace exhibiting a jagged appearance. When the launched pulses have a pulse width of $W$ and optical frequency of $v$, the backscattered wave at the input end at time $t_{0}$ will be the coherent summation of the fields backscattered from $N$ scatterers $[17,18]$ :

$$
E\left(t=t_{0}, z=0\right)=E_{0} \exp (-2 \alpha \bar{z}) \exp \left(j 2 \pi v t_{0}\right) \sum_{i=1}^{N} r_{i} \exp \left(j \varphi_{i}\right)
$$

where $\alpha$ is the optical fiber attenuation constant, $r_{i}$ and $\varphi_{i}$ are the reflectivity of the $i$ th scatterer and relative phase of the reflected wave, respectively. They are random parameters and uniformly distributed over $[0,1]$ and $[0,2 \pi]$, respectively. The fields interact with each other when they spatially overlap, corresponding to the half pulse width. Therefore, $\bar{z}$ defines the positions of the scatterers inside the half pulse width, i.e. $W / 2$, region, and expressed by $\left[t_{0} c / n_{f}-W / 2\right] / 2$. Here $c$ is the velocity of light in vacuum, and $n_{f}$ is the refractive index of the fiber.

The only random parameter, which affects the statistics of the signal and contributes to fading, is $\sum_{i=1}^{N} r_{i} \exp \left(j \varphi_{i}\right)$ [17]. For $\varphi$-OTDR systems, the backscattered signal intensity is observed, which is proportional to the square of the electric field. The intensity of the backscattered signal can be expressed as:

$$
\begin{aligned}
I & =\left|\exp \left(j 2 \pi v t_{0}\right)\right|^{2}\left|\sum_{i=1}^{N} r_{i} \exp \left(j \varphi_{i}\right)\right|^{2} \\
& =\left|\sum_{i=1}^{N} r_{i} \exp \left(j \varphi_{i}\right)\right|^{2} \\
& =\sum_{i=1}^{N} r_{i}^{2}+\sum_{j=1}^{N-1} \sum_{k=j+1}^{N} r_{j} r_{k} \cos \left(\varphi_{j}-\varphi_{k}\right)
\end{aligned}
$$

where the last term describes the multipoint interference between the scattered light from numerous scatterers within the half pulse width and results in a speckle like time domain pattern of the $\varphi$-OTDR trace.

If a perturbation is applied in the $q$ th scatterer among $N$ scatterers, which introduces a phase difference $\theta$, then the corresponding expression for the intensity, $\Delta I$, will be given by the intensity difference between two consecutive traces with and without perturbation:

$$
\Delta I=I_{\text {perturbed }}-I_{\text {non-perturbed }}=2 \sum_{j=1}^{q-1} \sum_{k=q}^{N} r_{j} r_{k}\left[\cos \left(\varphi_{j}-\varphi_{k}\right)-\cos \left(\varphi_{k}-\varphi_{j}-\theta\right)\right]
$$

This final expression models the non-linear response and fading behavior of the system. 

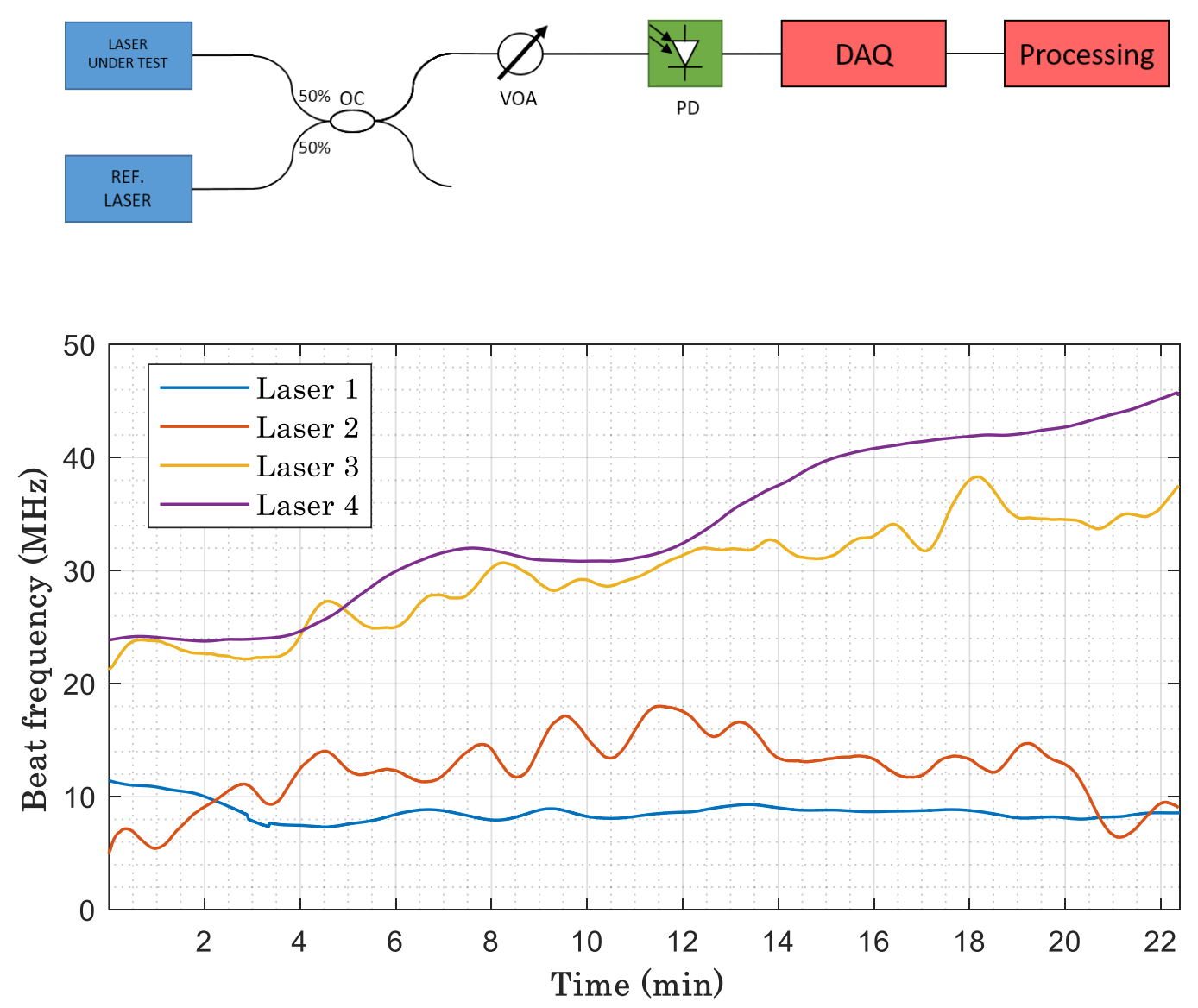


\begin{tabular}{|l|l|l|}
\hline & & \\
\hline & & \\
\hline & & \\
\hline & & \\
\hline & & \\
\hline
\end{tabular}
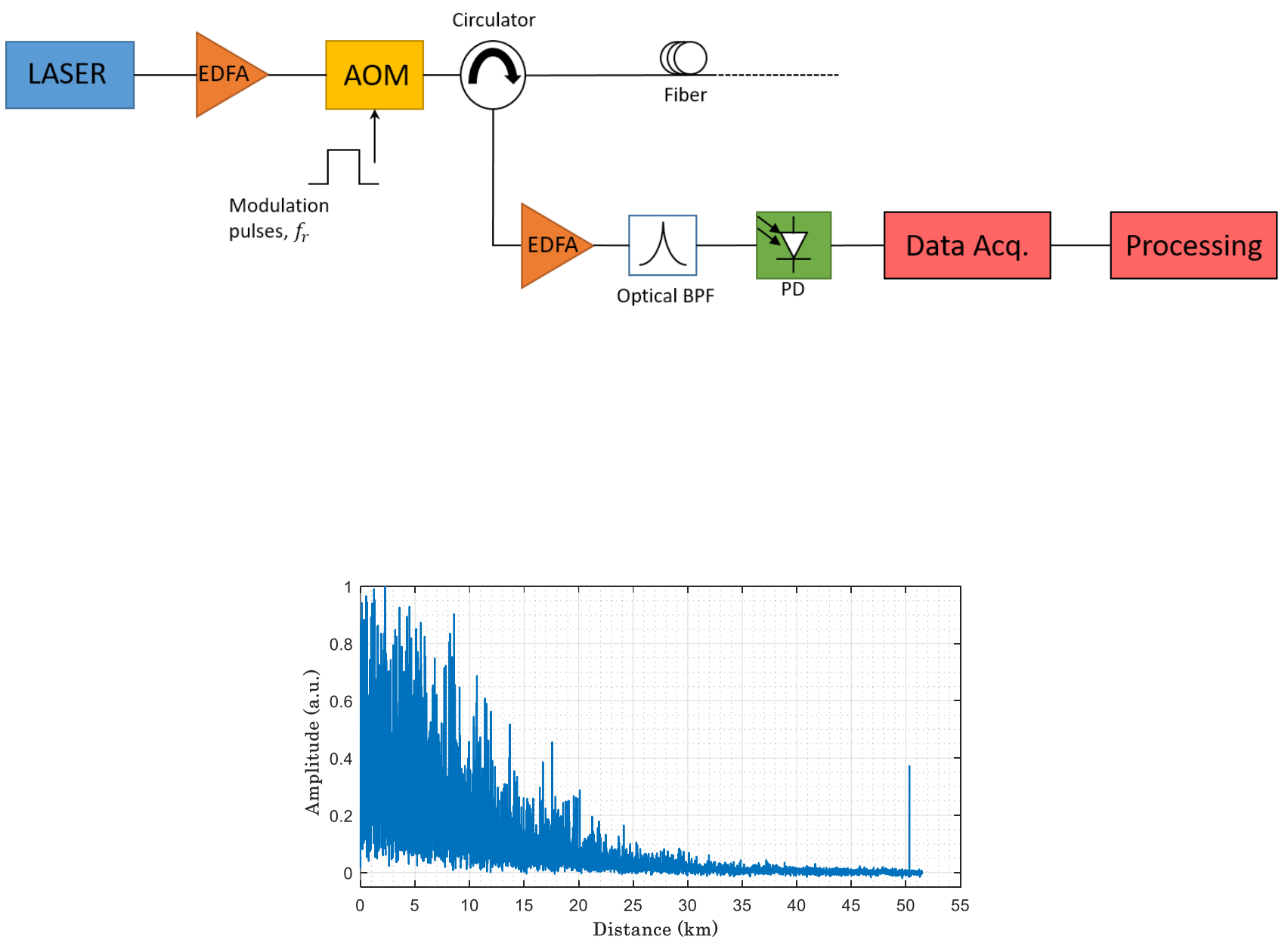

Proc. of SPIE Vol. 10654 106540D-4 


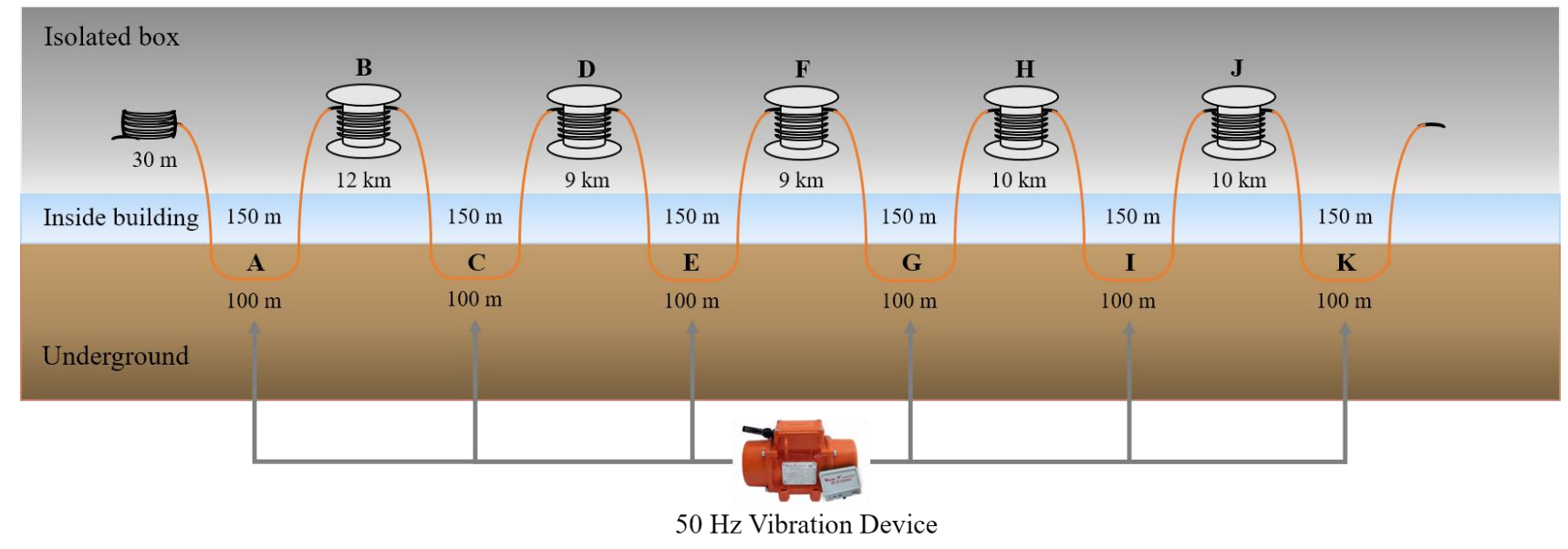




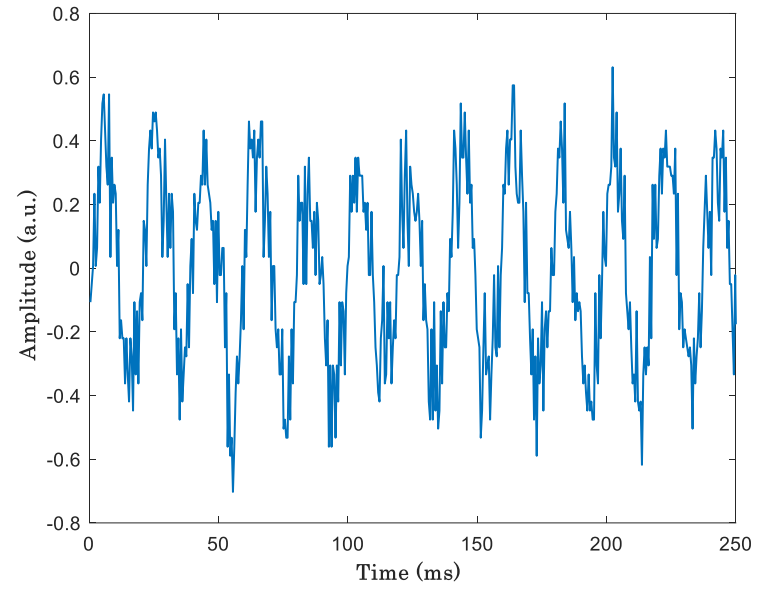

(a)

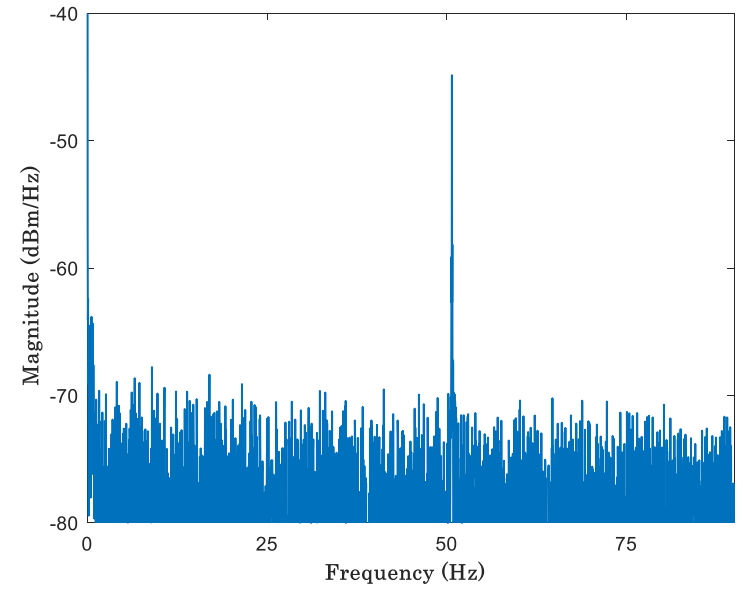

(b)

Figure 5. (a)Time trace and (b) spectrum of a $50 \mathrm{~Hz}$ vibration signal at $21 \mathrm{~km}$.
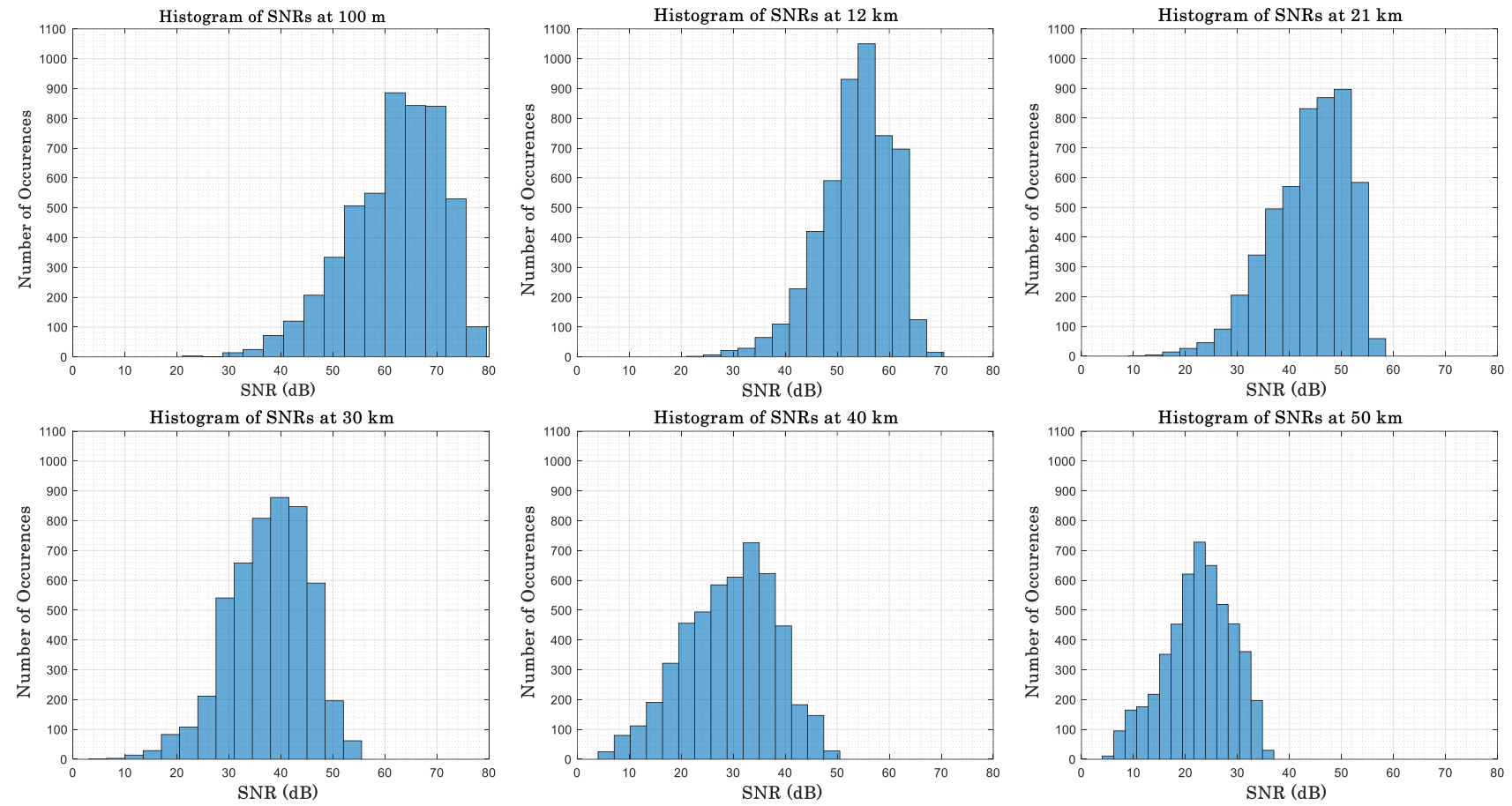

Figure 6. Histograms of SNRs at distances $100 \mathrm{~m}, 12 \mathrm{~km}, 21 \mathrm{~km}, 30 \mathrm{~km}, 40 \mathrm{~km}$ and $50 \mathrm{~km}$.

The mean SNR values show a linear decrease for longer distances as expected (Fig. 7). The decrease is around $8 \mathrm{~dB} / 10 \mathrm{~km}$ (in electronic domain), which is consistent with a fiber loss of $0.2 \mathrm{~dB} / \mathrm{km}$ (in optical domain). 


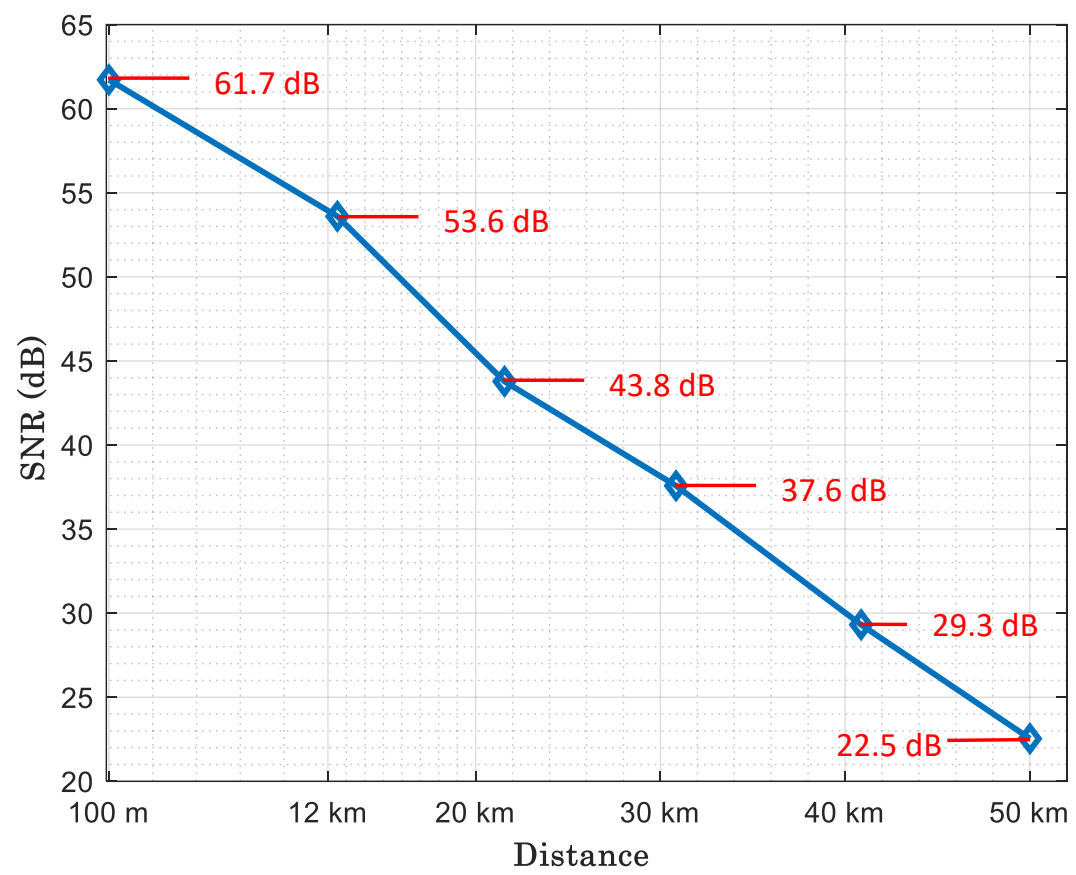

Figure 7. Mean SNR values in 6 different regions. It shows a linear decrease in $\mathrm{dB}$ scale and the system has a performance of $22.5 \mathrm{~dB}$ at $50 \mathrm{~km}$.

\section{CONCLUSION}

In this paper, we have presented the field demonstration of $\varphi$-OTDR based direct detection DAS system. A narrow linewidth and low frequency drift light source was preferred for a better performance. The intensities of vibration signal over the monitored regions were measured and their SNRs within certain time windows $(33.75 \mathrm{sec})$ were calculated. The resulting SNR values were used to generate corresponding histograms in 6 different locations from $100 \mathrm{~m}$ to $50 \mathrm{~km}$ along the fiber. Mean SNR values were calculated over these histograms and shown to linearly decrease with the distance as expected. The $\varphi$-OTDR based DAS system has a performance of $61.7 \mathrm{~dB}$ at the $100 \mathrm{~m}$ position and $22.5 \mathrm{~dB}$ at the $50 \mathrm{~km}$ position.

\section{REFERENCES}

[1] Liu, X., Jin, B., Bai, Q., Wang, Y., Wang, D., Wang, Y., "Distributed Fiber-Optic Sensors for Vibration Detection," Sensors 16(12), 1164 (2016)

[2] Lu, Y., Zhu, T., Chen, L., Bao, X., "Distributed Vibration Sensor Based on Coherent Detection of Phase-OTDR," Journal of Lightwave Technology (2010).

[3] Wang, Z. N., Zeng, J. J., Li, J., Fan, M. Q., Wu, H., Peng, F., Zhang, L., Zhou, Y., Rao, Y. J., "Ultra-long phasesensitive OTDR with hybrid distributed amplification," Optics Letters 39(20), 5866 (2014).

[4] Martins, H. F., Martin-Lopez, S., Corredera, P., Filograno, M. L., Frazao, O., Gonzalez-Herraez, M., "Phasesensitive Optical Time Domain Reflectometer Assisted by First-order Raman Amplification for Distributed Vibration Sensing Over >100 km," Journal of Lightwave Technology 32(8), 1510-1518 (2014).

[5] Fang, G., Xu, T., Feng, S., Li, F., "Phase-Sensitive Optical Time Domain Reflectometer Based on PhaseGenerated Carrier Algorithm," Journal of Lightwave Technology 33(13), 2811-2816 (2015).

[6] Masoudi, A., Belal, M., Newson, T. P., “A distributed optical fibre dynamic strain sensor based on phase-OTDR,” Measurement Science and Technology 24(8), 085204 (2013).

[7] Chen, D., Liu, Q., Fan, X., He, Z., "Distributed Fiber-Optic Acoustic Sensor With Enhanced Response Bandwidth and High Signal-to-Noise Ratio," Journal of Lightwave Technology 35(10), 2037-2043 (2017). 
[8] Pan, Z., Liang, K., Ye, Q., Cai, H., Qu, R., Fang, Z., "Phase-sensitive OTDR system based on digital coherent detection," Optical Sensors and Biophotonics (2011).

[9] Qin, Z., Zhu, T., Chen, L., Bao, X., "High Sensitivity Distributed Vibration Sensor Based on PolarizationMaintaining Configurations of Phase-OTDR," IEEE Photonics Technology Letters 23(15), 1091-1093 (2011).

[10] Shimizu, K., Horiguchi, T., Koyamada, Y., "Characteristics and reduction of coherent fading noise in Rayleigh backscattering measurement for optical fibers and components," Journal of Lightwave Technology 10(7), 982987 (1992).

[11] Shi, Y., Feng, H., Zeng, Z., “A Long Distance Phase-Sensitive Optical Time Domain Reflectometer with Simple Structure and High Locating Accuracy," Sensors 15(9), 21957-21970 (2015).

[12] Pan, Z., Liang, K., Zhou, J., Ye, Q., Haiwen Cai, Ronghui Qu, "Interference-fading-free phase-demodulated OTDR system", Proc. SPIE 8421, OFS2012 22nd International Conference on Optical Fiber Sensors, 842129 (2012)

[13] Ren, M., Lu, P., Chen, L., Bao, X., "Theoretical and Experimental Analysis of $\phi$-OTDR Based on Polarization Diversity Detection," IEEE Photonics Technology Letters 28(6), 697-700 (2016).

[14]Zhang, Y., Xu, Y., Shan, Y., Sun, Z., Zhu, F., Zhang, X., "Polarization dependence of phase-sensitive optical time-domain reflectometry and its suppression method based on orthogonal-state of polarization pulse pair," Optical Engineering 55(7), 074109 (2016).

[15] Gabai, H., Eyal, A., "SNR characterization in distributed acoustic sensing", Proc. SPIE 9916, Sixth European Workshop on Optical Fibre Sensors, 99162W (2016)

[16]Eyal, A., Gabai, H., Shpatz, I., "Distributed acoustic sensing: how to make the best out of the Rayleighbackscattered energy?," 25th International Conference on Optical Fiber Sensors (2017).

[17] Wojcik A., "Signal statistics of phase dependent optical time domain reflectometer," Ph.D. dissertation<http://oaktrust.library.tamu.edu/bitstream/handle/1969.1/4873/etd-tamu-2006C-ELEN-

Wojcik.pdf?sequence=1 > (17 March 2018 ).

[18] Martins, H. F., Martin-Lopez, S., Corredera, P., Filograno, M. L., Frazao, O., Gonzalez-Herraez, M., “Coherent Noise Reduction in High Visibility Phase-Sensitive Optical Time Domain Reflectometer for Distributed Sensing of Ultrasonic Waves," Journal of Lightwave Technology 31(23), 3631-3637 (2013).

[19]Zhirnov, A., Fedorov, A., Stepanov, K., Nesterov, E., Karasik, V., Svelto, C., and Pnev, A., "Effects of laser frequency drift in phase-sensitive optical time-domain reflectometry fiber sensors," ArXiv e-prints (Apr. 2016).

[20]Zhong, X., Zhang, C., Li, L., Liang, S., Li, Q., Lü, Q., Ding, X., Cao, Q., "Influences of laser source on phasesensitivity optical time-domain reflectometer-based distributed intrusion sensor," Applied Optics 53(21), 4645 (2014). 